included: atrial inhibited (AAI); ventricular inhibited (VVI-RV); dual chamber (DDD-RV); biventricular (DDD-BiV) and left ventricular pacing (DDD-LV). Base rate for pacing was set at $86 / \mathrm{min}$ and continued for the duration of level 3 care. The haemodynamic data were measured using a pulmonary arterial catheter using either thermodilution or continuous cardiac output measurements.

Results The baseline demographics are illustrated in Abstract 002 table 1 . The acute haemodynamic measurements at $18 \mathrm{~h}$, Abstract 002 table 2.

Abstract 002 Table 1

\begin{tabular}{llll}
\hline Demographic & $\begin{array}{l}\text { Biventricular } \\
\text { pacing }\end{array}$ & $\begin{array}{l}\text { Standard } \\
\text { pacing }\end{array}$ & p Value \\
\hline Age/years (SD) & $69.2(9.3)$ & $65.5(12.7)$ & 0.32 \\
Male gender/\% & 79 & 79 & 1.0 \\
NYHA score pre-operation (SD) & $2.5(0.7)$ & $2.4(0.9)$ & 0.69 \\
Euroscore/additive (SD) & $8.3(3.1)$ & $7(2.8)$ & 0.18 \\
QRS duration/ms (SD) & $113.3(29.3)$ & $115.2(24.4)$ & 0.84 \\
CABG only/\% & 63 & 58 & 0.63 \\
Ejection fraction (pre-op)/\% (SD) & $26.1(5.4)$ & $28.0(7.3)$ & 0.34 \\
Cardiopulmonary bypass time/min (SD) & $127.1(41.1)$ & $144(45.9)$ & 0.25 \\
Duration of Level 3 Care/hours (SD) & $44.5(36.8)$ & $57.1(73.0)$ & 0.56 \\
\hline
\end{tabular}

Abstract 002 Table 2

\begin{tabular}{lll}
\hline Pacing mode & $\begin{array}{l}\text { Cardiac output } \\
\text { l/min/(SD) }\end{array}$ & $\begin{array}{l}\text { p Value (paired } \\
\text { student t test vs AAI) }\end{array}$ \\
\hline AAI & $5.4(1.1)$ & \\
VVI & $4.3(1.7)$ & $<0.001$ \\
DDD-RV & $5.3(1.1)$ & 0.77 \\
DDD-BIV & $5.8(1.2)$ & 0.02 \\
DDD-LV & $5.4(0.7)$ & 0.73 \\
\hline
\end{tabular}

Conclusions BiV pacing significantly improved haemodynamics in the early part of the post-operative period compared to standard AAI pacing at $18 \mathrm{~h}$. There is a suggestion that the improvement in haemodynamic function may translate into clinical benefit. The duration of level 3 care was $57.1 \mathrm{~h}$ in the standard pacing group compared to $44.5 \mathrm{~h}$ in the $\mathrm{BiV}$ group. However, the $22 \%$ reduction in level 3 care in the BiV group compared to the standard pacing group did not reach statistical significance.

\section{VALIDATING MARKERS OF MECHANICAL DYSSYNCHRONY BY EXPERIMENTAL MANIPULATION OF INTERVENTRICULAR TIMINGS: WHAT IS NEEDED TO MAKE THEM A REASONABLE PROSPECT FOR CARDIAC RESYNCHRONISATION THERAPY SELECTION?}

doi:10.1136/heartjnl-2012-301877b.3

P A Pabari, ${ }^{*}$ M Moraldo, A D Hughes, J Mayet, A Kyriacou, D P Francis, C H Manisty. International Centre for Circulatory Health, Imperial College, London, UK

Background Any dyssynchrony marker proposed for selection of patients for cardiac resynchronisation therapy (CRT) should be stable between heartbeats but change markedly when interventricular delay is experimentally manipulated; the marker should also minimise at some "optimal" interventricular delay.

Methods and Results We performed 3264 echocardiographic measurements in 13 patients with CRT: separate, replicate measurements at interventricular delays from RV-first $40 \mathrm{~ms}$ to LV-first $60 \mathrm{~ms}$, in 20-ms intervals of (1) 3D systolic dyssynchrony index (SDI), (2) Tissue Doppler imaging (TDI), (3) aortic pre-ejection time, (4) interventricular mechanical delay (IVMD), (5) LVOT VTI and (6) ORS duration. In each patient, we identified with blinding on several successive repetitions, an apparently-optimal (minimallydyssynchronous) interventricular delay for each variable. Agreement between successive optimisations was low: $\kappa$ values of 0.24 for SDI, 0.02 for TDI, 0.36 for aortic pre-ejection time, 0.14 for IVMD, 0.40 for LVOT VTI and 0.47 for QRS duration. Intraclass correlation coefficient, indicating measurement reproducibility, was low when single measurements were taken (ranging across methods from 0.32 to 0.63 ), but improved when pairs of measurements were averaged (0.51 to $0.74, p=0.0008$ ). Using averages of pairs of measurements reduced the disagreement between replicate optimisations $p=0.007$. Conclusions Under blinded conditions these mechanical dyssynchrony markers cannot reliably discriminate even large changes in interventricular delay, and can be quickly rejected as candidates for predicting clinical benefit from CRT. It would save time and expense if markers considered for clinical trialling under formal scientific conditions first underwent screening for plausibility by such a stage of inexpensive, active experimentation.

\section{SIMULTANEOUS INVASIVE PRESSURE AND FLOW MEASUREMENTS DURING ATRIOVENTRICULAR DELAY IMPROVEMENT REVEAL A COMPENSATORY PERIPHERAL VASODILATOR RESPONSE WHICH ATTENUATES THE INITIAL BLOOD PRESSURE INCREMENT: IMPLICATIONS FOR THE DESIGN OF OPTIMISATION PROTOCOLS}

doi:10.1136/heartjnl-2012-301877b.4

${ }^{1} \mathrm{P}$ A Pabari, ${ }^{1} \mathrm{~A}$ Kyriacou, ${ }^{2} \mathrm{~W}$ Wright, ${ }^{1} \mathrm{~A} \mathrm{D}$ Hughes, ${ }^{1} \mathrm{~J}$ Mayet, ${ }^{1} \mathrm{Z}$ I Whinnett, ${ }^{1} \mathrm{D}$ P Francis, ${ }^{1} \mathrm{C} \mathrm{H}$ Manisty. ${ }^{1}$ International Centre for Circulatory Health, Imperial College, London, UK; ${ }^{2}$ Imperial College NHS Trust, London, UK

Introduction With synchrony of ventricular contraction already restored by cardiac resynchronisation therapy (CRT), optimisation of atrioventricular (AV) delay relies on improving filling. Although when AV delay is improved blood pressure immediately rises, there is a subsequent partial decline. Is this secondary decline because (1) non-invasive measurements are unreliable, (2) cardiac function increment is short-lived or (3) peripheral vasodilatation occurs? We conducted invasive experiments to distinguish between these hypotheses.

Methods Nine patients with heart failure and CRT underwent changes in programmed AV delay from $40 \mathrm{~ms}$ to $120 \mathrm{~ms}$. We simultaneously measured beat-by-beat invasive aortic pressure and flow, and non-invasive pressure (Finometer). Triplicate sets of experiments were performed and averaged to minimise the impact of noise.

Results There was an immediate increment in invasive blood pressure of $+14.7 \pm 2.0 \mathrm{~mm} \mathrm{Hg}(\mathrm{p}=0.0001)$, but after $\sim 10$ beats there

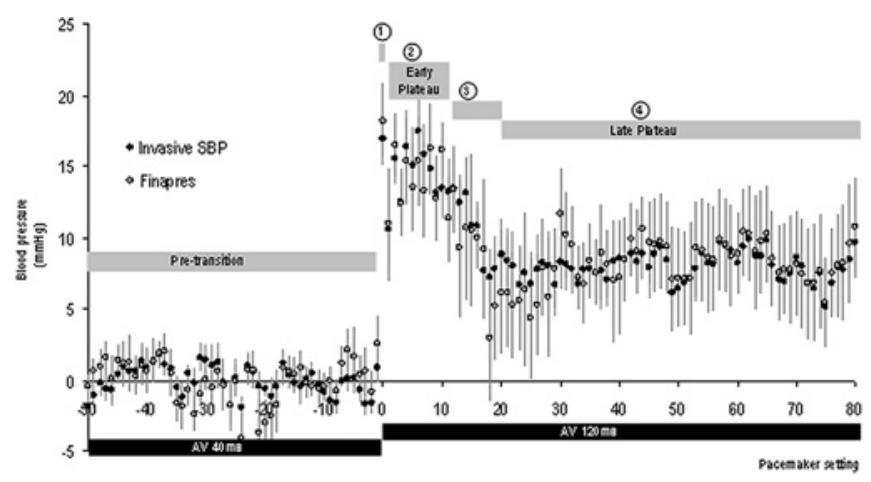

Abstract 004 Figure 1 